\title{
A NOTE ON AN EXAMPLE OF BIRMAN-MONTESINOS
}

\section{TAKESHI KANETO}

\begin{abstract}
We will report the negative solution to a conjecture of BirmanMontesinos about their example of Heegaard diagram. This implies the negative answer to a question of Waldhausen related to minimal Heegaard splittings of 3-manifolds.
\end{abstract}

We follow the notation and terminology of [1]. Let $(M, F ; v, w)$ be a Heegaard diagram and $(M, F)$ be the underlying Heegaard splitting. In [3] and [2] Waldhausen posed the following two questions:

Question A [3]. Suppose that $(M, F)$ is not minimal and that $(M, F ; v, w)$ is pseudominimal (in the sense of [1]). Does $(M, F ; v, w)$ have a cancelling pair of handles?

Question B [2]. Is there a manifold which admits two minimal Heegaard splittings of different genus?

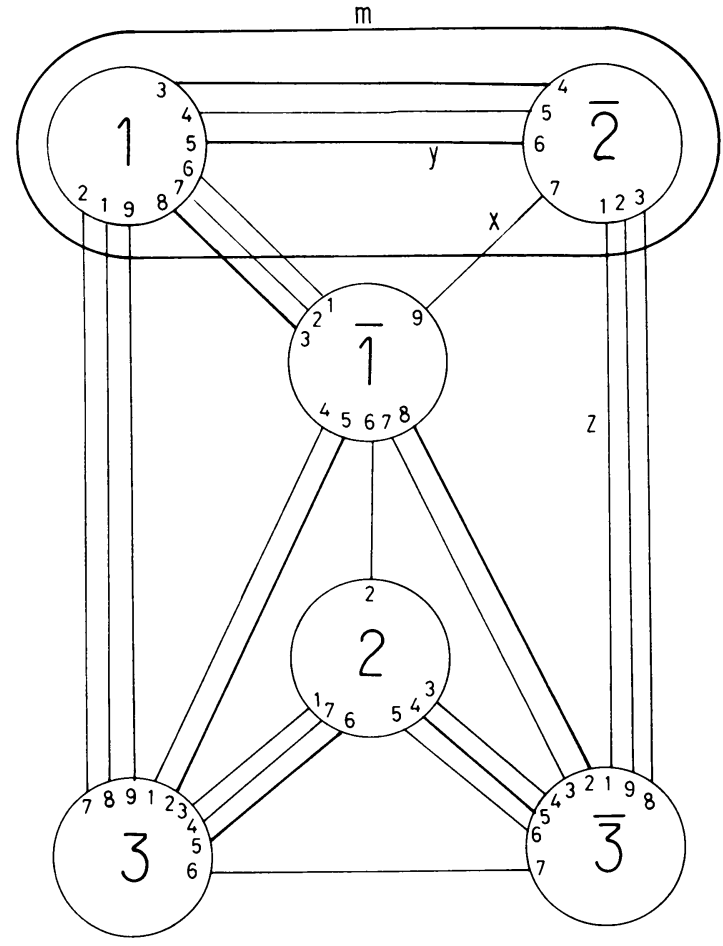

Received by the editors October 31, 1980.

1980 Mathematics Subject Classification. Primary 57A10.

Key words and phrases. 3-manifolds, minimal Heegaard splittings, pseudominimal Heegaard diagrams. 
Birman and Montesinos gave a delicate example in [1] which relates to these questions: their example is a pseudominimal Heegaard diagram without any cancelling pair of handles which is an affirmtive answer to Question B if the underlying Heegaard splitting is minimal, or a negative one to Question $A$ if it is not minimal. They conjectured that the former is the case. However, in this note we will show that the latter is the case. Let $(M, F ; v, w)$ be the Heegaard diagram of Birman-Montesinos, shown above (see Figure 10 of [1]) where $v=(1,2,3)$ and $w=(x, y, z)$.

Let $m$ be a meridian disk in the handlebody $V$ with the complete system $v$ of meridian disks as shown in the above figure. It is easy to see that $\partial m$ intersects $\partial y$ transversally at one point. Therefore, the Heegaard splitting $(M, F)$ is not minimal. Hence the Birman-Montesinos example gives a pseudominimal Heegaard diagram which has no cancelling pair of handles, although the underlying splitting $(M, F)$ is not minimal. Thus it gives a negative answer to Question A.

\section{REFERENCES}

1. J. S. Birman and J. M. Montesinos, On minimal Heegaard splittings, Michigan Math. J. 27 (1980), 49-57.

2. F. Waldhausen, Heegaard-Zerlegungen der 3-Sphäre, Topology 7 (1968), 195-203.

3. __ Some problems on 3-manifolds, Proc. Sympos. Pure Math., vol. 32, Amer. Math. Soc., Providence, R.I., 1978, pp. 313-322.

Department of Mathematics, University of Tsukuba, Ibaraki, JaPan 